\title{
The Potassium Channel KCa3.1 Represents a Valid Pharmacological Target for Astrogliosis-Induced Neuronal Impairment in a Mouse Model of Alzheimer's Disease
}

\author{
Tianjiao Wei ${ }^{1+}$, Mengni Yit ${ }^{1+}$, Wen $\mathrm{Gu}^{2 \dagger}$, Lina Hou ${ }^{1}$, Qin $\mathrm{Lu}^{1}$, Zhihua Yu ${ }^{1 *}$ and \\ Hongzhuan Chen ${ }^{1 *}$
}

'Department of Pharmacology, Institute of Medical Sciences, Shanghai Jiao Tong University School of Medicine, Shanghai, China, ${ }^{2}$ Department of Respiratory Medicine, Xinhua Hospital, Shanghai Jiao Tong University School of Medicine, Shanghai, China

\section{OPEN ACCESS}

Edited by:

Ashok Kumar,

University of Florida, USA

Reviewed by:

Takashi Kurihara,

Kagoshima University, Japan

Min-Yu Sun,

Washington University in St. Louis,

USA

${ }^{*}$ Correspondence:

Zhihua Yu

yuzhihua@shsmu.edu.cn

Hongzhuan Chen

hongzhuan_chen@hotmail.com

${ }^{t}$ These authors have contributed equally to this work.

Specialty section:

This article was submitted to

Neuropharmacology,

a section of the journal

Frontiers in Pharmacology

Received: 17 October 2016 Accepted: 20 December 2016 Published: 05 January 2017

Citation:

Wei T, Yi M, Gu W, Hou L, Lu Q

Yu Z and Chen H (2017)

The Potassium Channel KCa3.1

Represents a Valid Pharmacological

Target for Astrogliosis-Induced

Neuronal Impairment in a Mouse

Model of Alzheimer's Disease.

Front. Pharmacol. 7:528.

doi: 10.3389/fphar.2016.00528
Alzheimer's disease (AD) is a neurodegenerative disorder characterized by progressive decline of cognitive function. Astrogliosis plays a critical role in AD by instigating neuroinflammation, which leads ultimately to cognition decline. We previously showed that the intermediate-conductance $\mathrm{Ca}^{2+}$-activated potassium channel (KCa3.1) is involved in astrogliosis-induced by TGF- $\beta$ in vitro. In the present study, we investigated the contribution of KCa3.1 channels to astrogliosis-mediated neuroinflammation, using TgAPP/PS1 mice as a model for AD. We found that KCa3.1 expression was increased in reactive astrocytes as well as in neurons in the brains of both $\mathrm{Tg}^{\mathrm{APP}} / \mathrm{PS} 1$ mice and AD patients. Pharmacological blockade of KCa3.1 significantly reduced astrogliosis, microglial activation, neuronal loss, and memory deficits. KCa3.1 blockade inhibited astrocyte activation and reduced brain levels of $\mathrm{IL}-1 \beta$, TNF- $\alpha$, iNOS, and COX2. Furthermore, we used primary co-cultures of cortical neurons and astrocytes to demonstrate an important role for $\mathrm{KCa} 3.1$ in the process of astrogliosis-induced neuroinflammatory responses during amyloid- $\beta(A \beta)$-induced neuronal loss. KCa3.1 was found to be involved in the $A \beta$-induced activated biochemical profile of reactive astrocytes, which included activation of JNK MAPK and production of reactive oxygen species. Pharmacological blockade of KCa3.1 attenuated $A \beta$-induced reactive astrocytes and indirect, astrogliosis-mediated damage to neurons. Our data clearly indicate a role for astrogliosis in $\mathrm{AD}$ pathogenesis and suggest that $\mathrm{KCa} 3.1$ inhibition might represent a good therapeutic target for the treatment of AD.

\section{Highlights:}

(1) Blockade of KCa3.1 in APP/PS1 transgenic mice attenuated astrogliosis and neuron loss, and an attenuation of memory deficits. (2) Blockade of KCa3.1 attenuated $A \beta$-induced indirect, astrogliosis-mediated damage to neurons in vitro via activation of JNK and ROS.

Keywords: astrogliosis, Alzheimer's disease, GFAP, inflammation, neurons

Abbreviations: $\mathrm{CM}-\mathrm{H}_{2}$ DCFDA, 5-(and-6)-chloromethyl-2', $7^{\prime}$-dichlorodihydrofluorescein diacetate; GFAP, glial fibrillary acidic protein; KCa3.1, intermediate-conductance calcium-activated potassium channel; TGF- $\beta$, transforming growth factor$\beta$; TRAM-34, 1-[(2-chlorophenyl)(diphenyl)methyl]-1H-pyrazole. 


\section{INTRODUCTION}

Alzheimer's disease (AD) is a neurodegenerative disorder characterized by progressive decline of cognitive function. The proposed mechanisms of cognitive impairment include synaptic dysfunction triggered by $\beta$-amyloid $(A \beta)$, neuronal death, oxidative stress, tau pathology, and glutamate excitotoxicity. It is widely acknowledged that reactive gliosis plays a significant role in the progression of $\mathrm{AD}$. Accumulation of reactive astrocytes and activation of microglia in affected brain regions are apparent in both $\mathrm{AD}$ patients and the majority of transgenic rodent models of AD. Garwood et al. (2011) reported that reactive astrogliosis is involved in regulating $A \beta$-induced neurotoxicity and tau phosphorylation. Reactive astrocytes release a variety of cytokines and pro-inflammatory mediators, which activate intracellular signaling pathways including extracellular signalingrelated kinase (ERK), c-Jun N-terminal kinase (JNK), protein kinase $\mathrm{C}$ and PI3 kinase (Matos et al., 2008).

It has been reported that brief (5-15 min) application of $A \beta$ to mixed disassociated hippocampal cultures evoked $\mathrm{Ca}^{2+}$ influx and up-regulated reactive oxygen species (ROS) in astrocytes, but not in neurons, whereas longer $(24 \mathrm{~h})$ exposure led to cell death, especially in neurons (Abramov et al., 2003). Although these data indicate that the primary target of $A \beta$ might be astrocytes and that $A \beta$-induced oxidative stress and calcium overload in astrocytes may lead to neuronal death, the mechanism of these effects is not well understood.

In immune cells, airway smooth muscle cells and fibroblasts, the intermediate-conductance $\mathrm{Ca}^{2+}$-activated potassium channel KCa3.1 regulates membrane potential through $\mathrm{K}^{+}$efflux, thus facilitating $\mathrm{Ca}^{2+}$ entry (Toyama et al., 2008; Di et al., 2010; Yu et al., 2013; Yi et al., 2016a). In the central nervous system (CNS), KCa3.1 plays a role in neuroprotection following traumatic brain injury, stroke and spinal cord injury (Bouhy et al., 2011). Up-regulation of KCa3.1 has been detected in reactive astrocytes in a mouse model of spinal cord injury. We have previously reported that blockade of KCa3.1, or gene deletion, attenuated TGF- $\beta$-induced astrogliosis by regulating intracellular $\mathrm{Ca}^{2+}$ in primary astrocytes (Yu et al., 2014). Yi et al. (2016a) reported that KCa3.1 is involved in scratch-induced migration of reactive astrocytes mediated by the JNK/c-Jun pathway. More recently, we demonstrated that blockade of $\mathrm{KCa} 3.1$ in senescenceaccelerated mouse prone 8 (SAMP8) mice resulted in a decrease in astrogliosis and, moreover, an attenuation of memory deficits (Yi et al., 2016b). Evidence was found for astrogliosis in AD patients with mild cognitive impairment in a positron emission tomography study (Carter et al., 2012). Most recently, Schöll et al. (2015) reported that measure of astrogliosis in autosomal dominant $\mathrm{AD}$ could be observed decades before symptom onset using multi-tracer positron emission tomography, possibly coinciding with early fibrillar $\mathrm{A} \beta$ plaque deposition. Although reactive astrogliosis has long been recognized as a pathological feature of $\mathrm{AD}$, the role of astrogliosis in the process of cognitive decline in $\mathrm{AD}$ is still poorly understood.

In the present study, we showed that blockade of KCa3.1 attenuated $A \beta$-induced changes in the biochemical profile of reactive astrocytes, including activation of JNK MAP kinase (MAPK) and production of ROS. An experimental KCa3.1 blocker, TRAM-34, attenuated $A \beta$-induced, indirect, astrogliosis-mediated damage to neurons in vitro, decreased astrogliosis and neuronal loss, and attenuated memory deficits in $\mathrm{APP}^{\text {Swe }} / \mathrm{PS1}^{\mathrm{A} 246 E}$ transgenic (Tg ${ }^{\mathrm{APP} / \mathrm{PS} 1}$ ) mice in vivo.

\section{MATERIALS AND METHODS}

\section{Brain Autopsy Material}

Human brain sections were collected from six $\mathrm{AD}$ cases and six control cases (Netherlands Institute for Neuroscience, Amsterdam, Netherlands). Written informed consent for a brain autopsy to be used for research purposes after death had been obtained by the Netherlands Brain Bank.

\section{Animals}

Nine-month male transgenic mice (the Jackson Lab, no. 003378, $\mathrm{Tg}^{\mathrm{APP} / \mathrm{PS} 1}$ ) were purchased from the Jackson lab. Mice were divided into the following three groups: littermate wild type (WT) mice $(n=20)$, transgenic (Tg) mice with vehicle treatment $(n=20)$ or TRAM-34 $(120 \mathrm{mg} / \mathrm{kg}$, intraperitoneal, Apptec, Wuxi, China) treatment (Tg + TRAM-34, $n=20)$. After 4 weeks of drug treatment, the mice were submitted to behavioral testing, and then the mice were euthanized and brain tissues were collected for Western blotting or histology analyses. The protocol of animal experiments was approved by the Animal Experimentation Ethics Committee of Shanghai Jiao Tong University School of Medicine.

\section{Morris Water Maze Test}

A modified Morris water maze test was carried out as previously described (Morris, 1984). The test requires the animals to find a visible or hidden platform in a large pool of opaque water and involves 5 days of training sessions ( 1 day with the platform visible and 4 days with the platform hidden), followed by a spatial probe trial, with no platform present, on day 6. During the hidden platform training, the mice were able to swim freely for $60 \mathrm{~s}$ to find a platform $1 \mathrm{~cm}$ below the water surface. During the spatial probe trial, the directness of the route taken to the area where the platform was previously located, together with the percentage of total time spent in this quadrant of the pool, was recorded using a video tracking system (Jiliang Software Technology Co., Ltd., Shanghai, China).

\section{Open Field Test}

The open field test was carried out as described previously ( $\mathrm{Wu}$ et al., 2016). Briefly, the mouse was gently placed in the center of an open field chamber $(40 \mathrm{~cm} \times 40 \mathrm{~cm} \times 40 \mathrm{~cm})$ and was allowed to move freely for $5 \mathrm{~min}$. The movement parameters of the mouse were monitored and analyzed via a video camera connected to a tracking system (Jiliang Software Technology Co., Ltd., Shanghai, China). After each test, the floor of the open field was cleaned with solution of $70 \%$ ethanol to hide animal clues. The ratios of distance and time in the center were measured. 


\section{Immunostaining and Data Analysis}

The mice were anesthetized with chloral hydrate and perfused with $4 \%$ paraformaldehyde. The brain tissues were collected and cryoprotected with $30 \%$ sucrose in $0.1 \mathrm{M}$ phosphate buffered saline (PBS). Brain sections (12 $\mu \mathrm{m}$ thick) were blocked with $3 \%$ bovine serum albumin in $0.1 \mathrm{M}$ PBS for $1 \mathrm{~h}$ at room temperature and were then incubated with the following primary antibodies: mouse anti-KCa3.1 (1:200, Santa Cruz), rabbit anti-GFAP (1:1000; DAKO, Glostrup, Denmark), rabbit anti-NeuN (1:100, Millipore), rabbit anti-Iba1 (1:500, Wako) at $4^{\circ} \mathrm{C}$ overnight. Brain sections were labeled with either Alexa Fluor 488- or 568-conjugated anti-mouse or rabbit IgG (1:1000, Invitrogen). A TCS SP8 confocal laser scanning microscope (Leica, Germany), equipped with an argon-ion laser source, was used to capture images using excitation wavelengths of 405, 488, and $568 \mathrm{~nm}$ for DAPI, Alexa 488 and Alexa 568, respectively. Using the same reference position for each brain slice, between three and five random $0.01 \mathrm{~mm}^{2}$ microscopic fields were selected for quantification. Quantification was carried out in six slices of each brain (120 $\mu \mathrm{m}$ intervals), using immunoreactivity for GFAP, $\mathrm{Ibal}$, and NeuN expression. The numbers of $\mathrm{GFAP}^{+}, \mathrm{Ibal}^{+}$, and $\mathrm{NeuN}^{+}$positive cells were counted in six slices per mouse in a blinded manner, using Leica LAS AF Lite software to measure the areas.

\section{Senile Plaque Staining}

For senile plaques staining, coronal sections $(12 \mu \mathrm{m})$ were cut using a Leica cryostat (Leica CM1850). Primary 6E10 antibody (SIG-39300, Covance, Princeton, NJ, USA) was used on the sections overnight at $4{ }^{\circ} \mathrm{C}$. Sections were incubated with biotinylated secondary antibody (AK-6602, Vector) for $45 \mathrm{~min}$. Sections were developed using the $A B C$ elite kit (AK-6600, Vector). Image-Pro plus software (Media cybernetics, USA) was used to measure and recorded as the average plaque areas per field. Six slices per mouse were used to count the plaque number in a blinded manner.

\section{Enzyme-Linked Immunosorbent Assay}

ELISA was performed using the kit for TNF- $\alpha$ and IL-1 $\beta$ (Rapidbio Labs, Langka Trade Co. Ltd., Shanghai, China). The procedures were conducted according to manufacturer's protocols.

\section{Preparation of Oligomeric $A \beta_{1-42}$ Peptides}

Our preparation of oligomeric amyloid $\beta$ (A $\beta 1-42)$ follows the procedure described previously (Wang et al., 2012). As described previously, monomeric peptide $A \beta 1-42$ was initially dissolved in 1,1,1,3,3,3-hexafluoro 2-propanol (HFIP, Sigma, St. Louis, MO, USA) at $1 \mathrm{mg} / \mathrm{ml}$. Dried peptides were resolved in dimethyl sulfoxide (DMSO), diluted to $100 \mu \mathrm{M}$ with $\mathrm{ddH}_{2} \mathrm{O}$, and incubated at $4^{\circ} \mathrm{C}$ for $24 \mathrm{~h}$.

\section{Primary Cultures}

Primary cortical astrocyte cultures derived from neonatal (P0P2) C57BL/6J mice were prepared from mixed glial cultures
(10-14 days in vitro) as described previously (Wang et al., 2008). Astrocyte-conditioned medium (ACM) for treating neurons was obtained using the following procedure. Firstly, astrocytes were cultured in Dulbecco's Modified Eagle's medium (DMEM) contained $10 \%$ fetal bovine serum. The culture medium (CM) was changed to neurobasal medium with B27 supplement (Invitrogen) after confluent astrocytes were serum-free for $24 \mathrm{~h}$. The NB/B27-based astrocytes were then treated with $A \beta_{1-42}$ oligomer $(5 \mu M)$ for different lengths of time. The $\mathrm{CM}$ from the NB/B27-based cells was collected and used immediately.

Neuronal cultures were established by adding cytosine arabinoside $(2 \mu \mathrm{M})$ to the mixed glial cultures to inhibit proliferation of glial cells. The neurons were cultured for at least 14 days before treatment with astrocytes CM.

Primary mixed cortical cells were isolated from neonatal (P0-P2) C57BL/6J mice and cultured as described previously (Rodriguez-Gonzalez et al., 2016). Briefly, astrocytes were collected and seeded in multiwell plates in DMEM. Four days after plating, neurons were obtained from brain cortices of $\mathrm{P} 0$ C57BL/6J mice and seeded on top of the astrocyte layer.

A Cell Counting Kit-8 (CCK-8, Dojindo Laboratories, Kumamoto, Japan) was used to measure cell viability (Yu et al., 2013).

\section{Neurite Outgrowth Assay}

$\mathrm{A} \beta_{1-42}$ oligomers were added to the primary cultured neurons with or without TRAM-34 and then stained with primary antibody microtubule associated protein 2 (MAP2) plus Alexa Fluor 555-conjugated secondary antibody and DAPI. Cellomics KineticScan reader was used to scan the MAP2 positive cells. Extended Neurite Outgrowth software (Thermo Scientific, Philadelphia, PA, USA) was used to analyze the data.

\section{Western Blot Analysis}

Protein extracts were separated by $10 \%$ (w/v) sodium dodecyl sulfate-polyacrylamide gel electrophoresis and were transferred to polyvinylidene difluoride membrane. The membrane was incubated with following primary antibodies for anti-total p38/JNK/ERK MAPK, anti-phospho-p38/JNK/ERK MAPK antibodies (1:1000, Cell Signaling Technology, Danvers, MA, USA), anti-Synaptophysin antibody (1:1000, Abcam), antiPSD95 antibody (1:1000, Abcam), anti-NeuN antibody (1:1000, Millipore), anti-MAP2 antibody (1:1000, Abcam), anti-GFAP antibody (1:5000, Dako, Glostrup, Denmark), anti-KCa3.1 antibody (1:400, Abcam), and anti- $\beta$-actin antibody (1:3000, Sigma). Secondary antibodies were horseradish peroxidaseconjugated antibody (1:3000; Amersham Biosciences) for $1 \mathrm{~h}$ at room temperature.

\section{Reactive Oxygen Species Measurement}

$\mathrm{A} \beta_{1-42}$ oligomers were added to the primary cultured astrocytes with or without TRAM-34. Astrocytes were loaded with $30 \mu \mathrm{M} 5-$ (and-6)-chloromethyl-2', $7^{\prime}$-dichlorodihydrofluorescein diacetate $\left(\mathrm{CM}-\mathrm{H}_{2} \mathrm{DCFDA}\right.$, Invitrogen, Waltham, CA, USA) to measure ROS generation as previously described (Alberdi et al., 2013). 


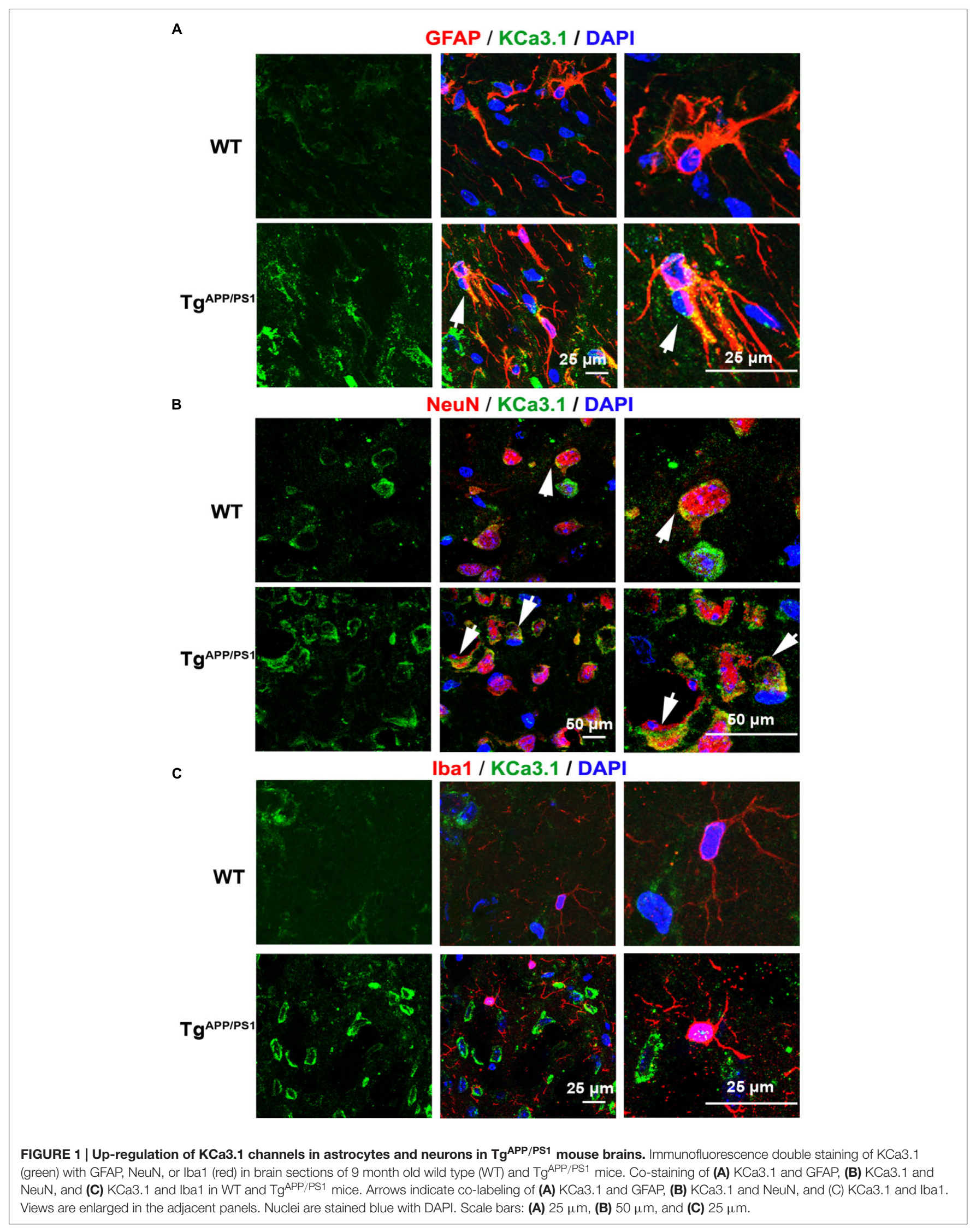




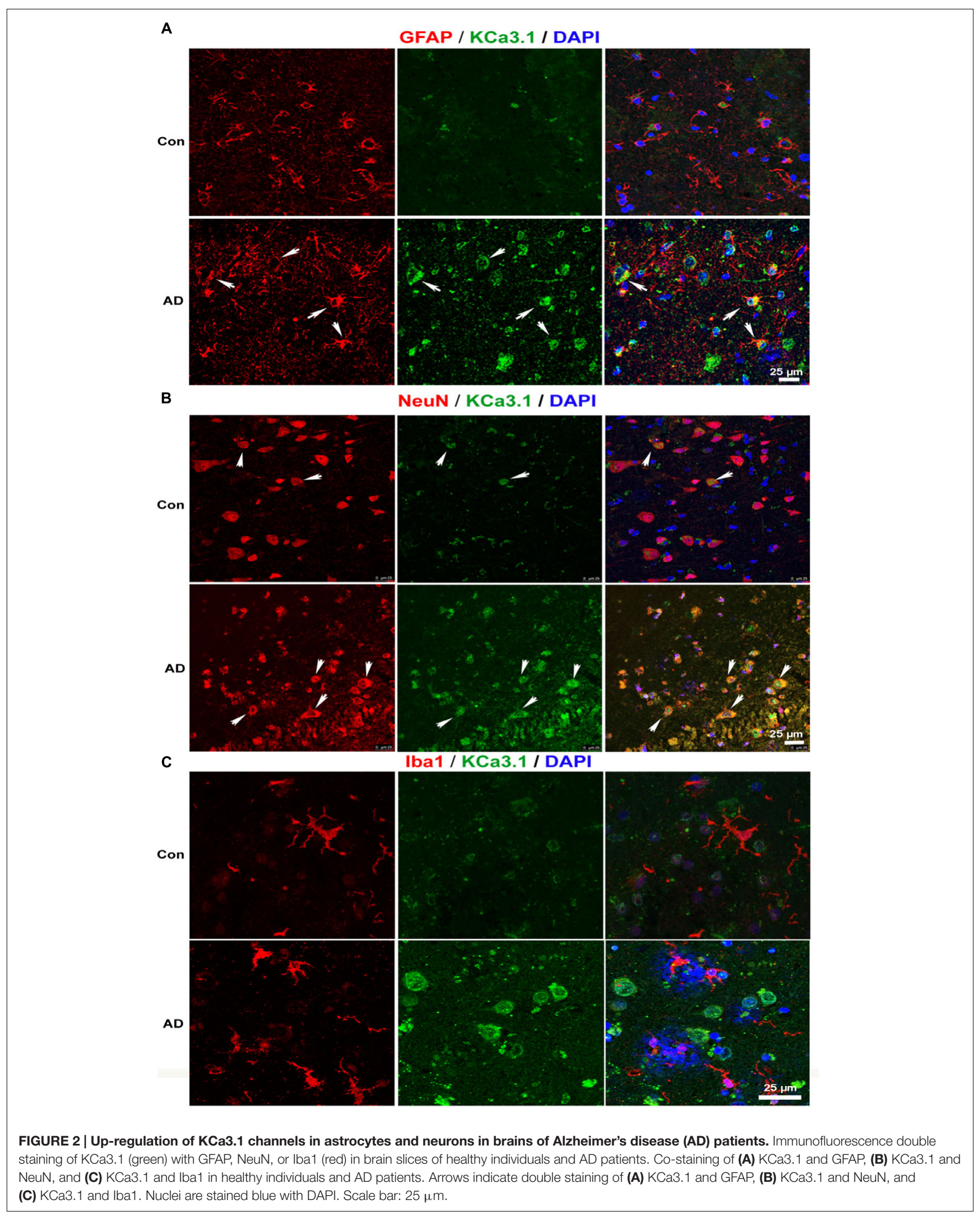




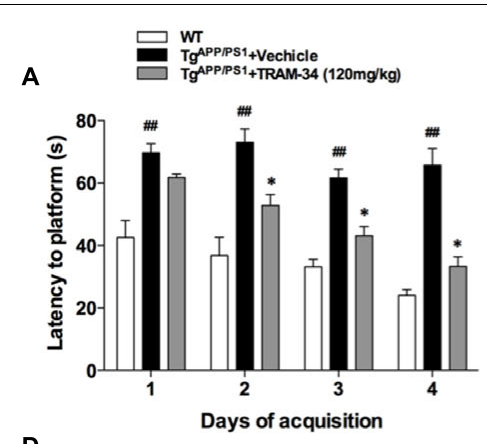

D

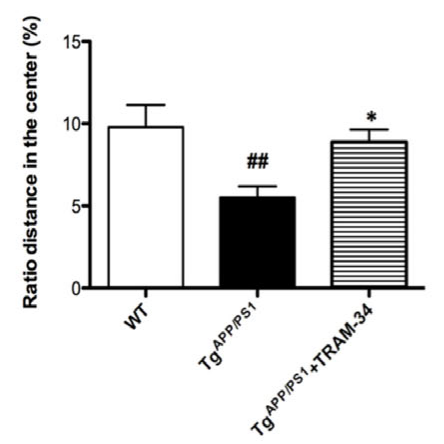

B

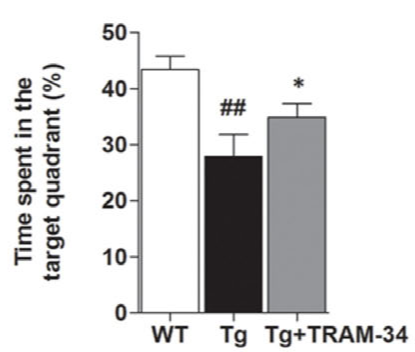

E

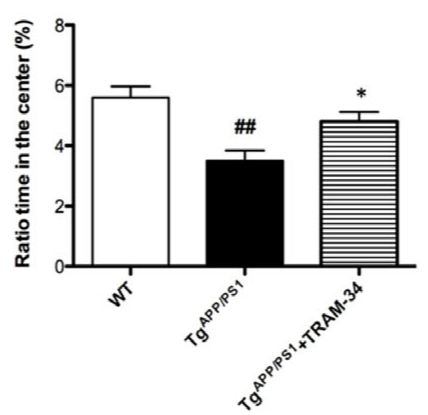

c

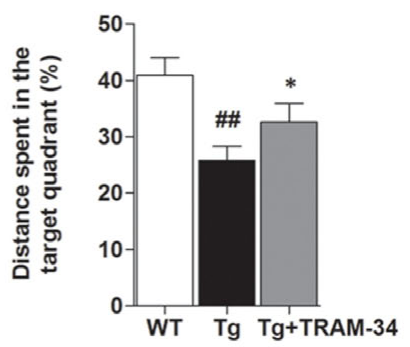

FIGURE 3 | Blockade of KCa3.1 rescued memory deficits and spontaneous motor activity in Tg ${ }^{\text {APP/PS1 }}$ mice. The Morris water maze test (A-C) and open field test (D,E) were performed on 9 months old mice as described in the section "Materials and Methods." TgAPP/PS1 mice were treated with vehicle or TRAM-34 (120 mg/kg, intraperitoneally) daily for 4 weeks. (A) Average latency to hidden platform, (B) Percentage of time spent by each group swimming in target quadrant during probe trial (no platform), (C) Percentage of total swim distance by each group in target quadrant during probe trial (no platform), (D,E) Exploratory locomotion of the open field test, (D) Ratio of distance in exploratory locomotion of the open field test, and (E) Ratio of time in center in exploratory locomotion of the open field test. TgAPP/PS1 group showed decreased locomotion compared with TgAPP/PS1 + TRAM-34 (120 mg/kg, intraperitoneally) group. Data represent mean \pm SEM ( $n=20$ per group). ${ }^{\# \#} p<0.01$ versus WT mice. ${ }^{*} p<0.05$ versus vehicle-treated TgAPP/PS1 mice. Tg, TgAPP/PS1.

\section{Statistical Analysis}

Statistical significance was analyzed using the Student's $t$ test, one-way ANOVA followed by Dunnett's post hoc tests or two way ANOVA followed by Bonferroni post hoc test, depending on the case, using Prism software (GraphPad Software, Inc., La Jolla, CA, USA). Results are presented as Mean \pm SEM, with $p<0.05$ considered to be statistically significant.

\section{RESULTS}

\section{KCa3.1 Is Up-Regulated in Reactive Astrocytes of $\mathrm{Tg}{ }^{\mathrm{APP}} / \mathrm{PS} 1$ Mice and AD Patients}

We recently showed that $\mathrm{KCa} 3.1$ expression is increased in both reactive astrocytes and neurons in the brains of SAMP8 mice, a model that is generally used to investigate the mechanisms of agerelated memory deficits (Yi et al., 2016b). In the present study, expression of KCa3.1 was detected in the brains of $\mathrm{Tg}^{\mathrm{APP} / \mathrm{PS} 1}$ mice at 9 months of age and in AD patients. Age-matched WT littermates and healthy humans were used as controls. In WT mice (Figure 1A) and healthy humans (Figure 2A), there was little co-localization between $\mathrm{KCa} 3.1$ and $\mathrm{GFAP}^{+}$ astrocytes. In 9 month old $\mathrm{Tg}^{\mathrm{APP} / \mathrm{PS} 1}$ mice (Figure 1A) and $\mathrm{AD}$ patients (Figure 2A), KCa3.1 was detected in $\mathrm{GFAP}^{+}$ hypertrophic astrogliosis. Kaushal et al. (2007) reported that $\mathrm{KCa} 3.1$ is expressed in rat neurons in vitro. In the present study, expression of KCa3.1 was shown to be higher in $\mathrm{NeuN}^{+}$neurons of $\mathrm{Tg}^{\mathrm{APP} / \mathrm{PS} 1}$ mice (Figure 1B) and AD patients (Figure $2 \mathbf{B}$ ) than in WT mice and control humans, where expression levels are low. Although there is convincing evidence for the expression of KCa3.1 on microglia in vitro, data supporting expression of KCa3.1 on microglia in vivo are scarce (Kaushal et al., 2007). In this study, rare co-localization of $\mathrm{KCa} 3.1$ and $\mathrm{Iba1}^{+}$microglia was detected in brain slices of both WT and $\mathrm{Tg}^{\mathrm{APP} / \mathrm{PS} 1}$ mice (Figure 1C) and control humans and AD patients (Figure 2C).

KCa3.1 was expressed at low levels, mainly in neurons, in WT mice and control humans. In $\mathrm{Tg}^{\mathrm{APP} / \mathrm{PS} 1}$ mice and $\mathrm{AD}$ patients, KCa3.1 expression was up-regulated in neurons and was also observed in astrocytes.

\section{Blockade of KCa3.1 Rescues Memory Deficits and Spontaneous Motor Activity in $\mathrm{Tg}$ APP/PS1 Mice}

In a previous study, we showed that inhibition of KCa3.1 channels attenuated memory deficits in SAMP8 mice (Yi et al., 2016b). In the present study, TRAM-34 (120 mg/kg, 


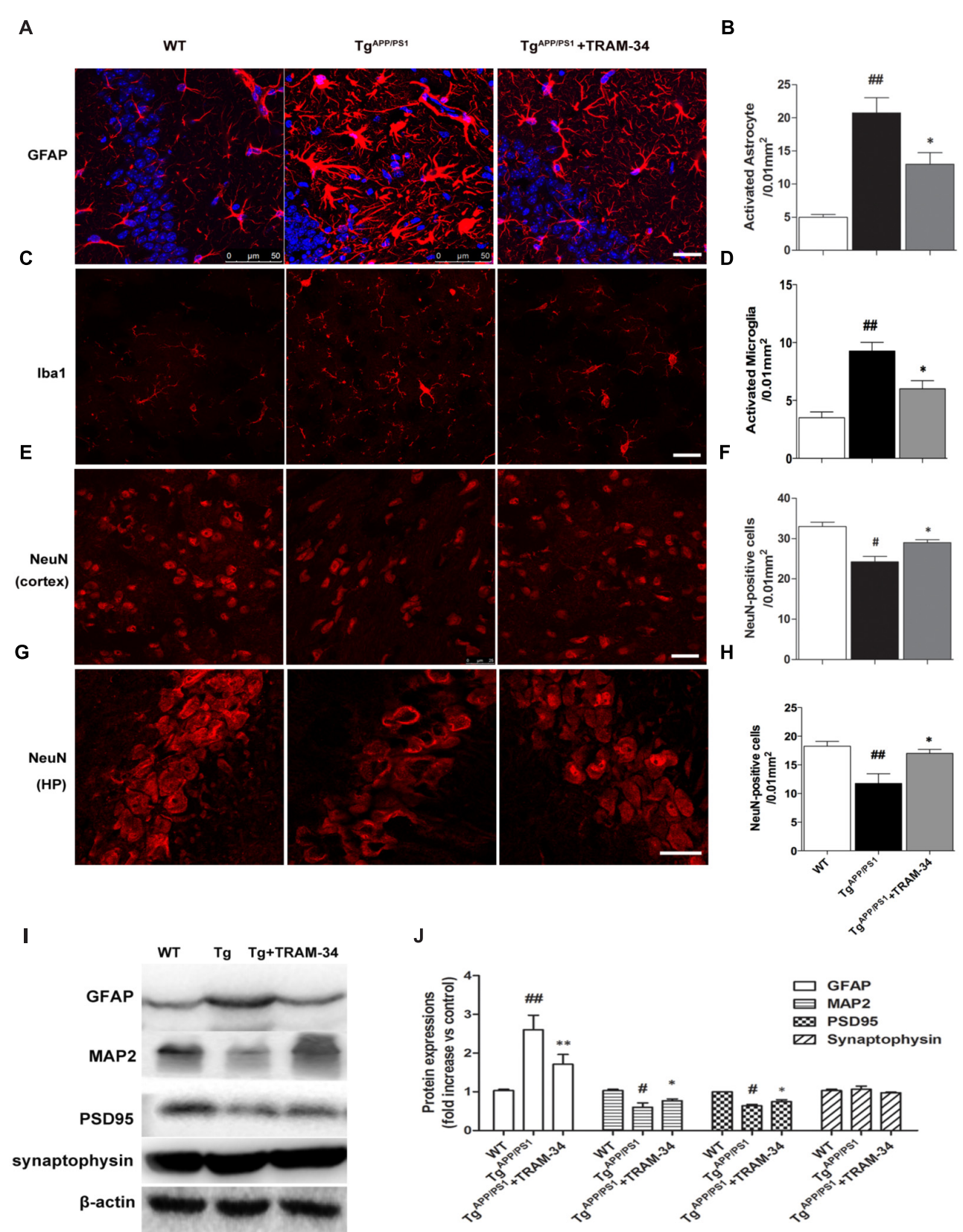

FIGURE 4 | Blockade of KCa3.1 attenuated gliosis and neuronal loss in TgAPP/PS1 mice. (A) Representative images of GFAP-immunoreactive astrocytes from hippocampal regions of WT and Tg mice, with and without TRAM-34 (120 mg/kg/d). (B) Quantification of reactive astrocyte number/0.01 mm² in hippocampus ( $n=6$-8). (C) Iba1 immunoreactivity of activated microglia from hippocampal regions of WT and Tg mice, with and without TRAM-34 (120 mg/kg/d).

(D) Quantification of activated microglial number/0.01 $\mathrm{mm}^{2}$ in hippocampus $(n=6-8)$. NeuN immunoreactivity of neurons in (E) cortex and (G) hippocampal regions of WT and Tg mice, with and without TRAM-34 (120 mg/kg/d). Quantification of neuron number/0.01 mm² in (F) cortex and (H) hippocampus $(n=6-8)$. Data represent mean \pm SEM. ${ }^{*} p<0.05,{ }^{\# \#} p<0.01$ compared with WT mice. ${ }^{*} p<0.05$, versus Tg mice $(n=6-8)$. (I) Western blot analysis using antibodies to astrocyte marker GFAP, dendritic protein MAP2, post-synaptic protein PSD95, and pre-synaptic protein synaptophysin. (J) Results are presented as mean \pm SEM $(n=4) .{ }^{\#} p<0.05,{ }^{\# \#} p<0.01$ compared with WT mice. ${ }^{*} p<0.05,{ }^{* *} p<0.01$ compared with Tg mice. Tg, TgAPP/PS1. Scale bar: $25 \mu \mathrm{m}$.

intraperitoneally) was used to test whether pharmacological blockade of KCa3.1 would attenuate loss of memory and spontaneous motor activity in $\mathrm{Tg}^{\mathrm{APP} / \mathrm{PS} 1}$ mice, and the dosage of TRAM-34 was used as previously described (Bouhy et al., 2011; Chen et al., 2016; Yi et al., 2016b). Nine months old $\mathrm{Tg}^{\mathrm{APP}}$ PS1 mice were treated once daily with TRAM34 (120 mg/kg, intraperitoneally) or vehicle for 4 weeks and the Morris water maze test and open field test were then used to assess memory and motor activity, respectively. In the hidden platform arm of the Morris water maze 

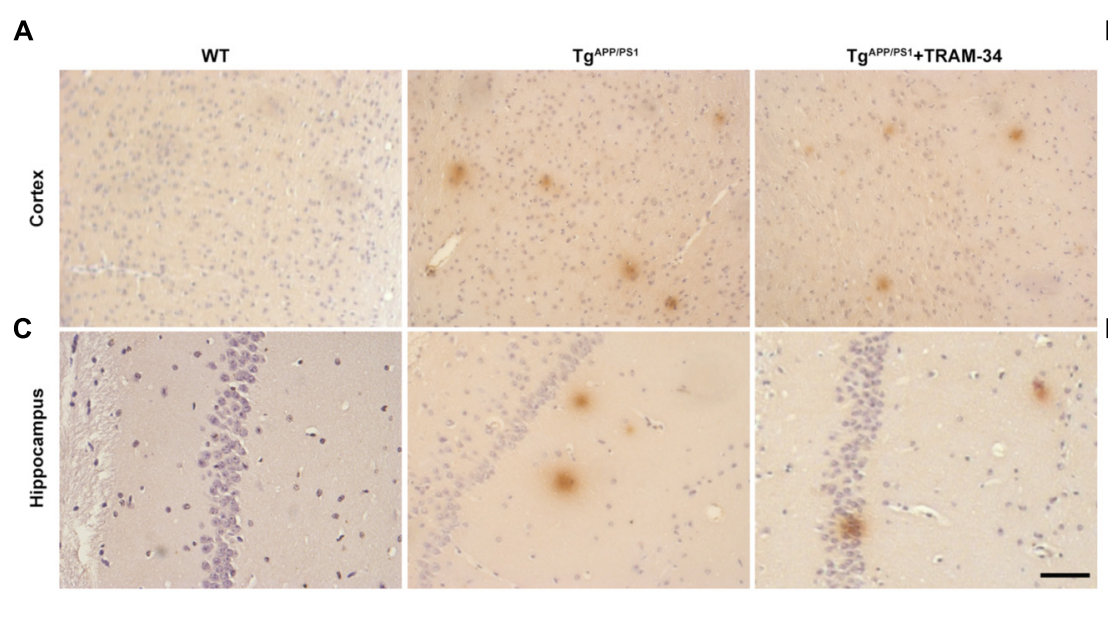

B
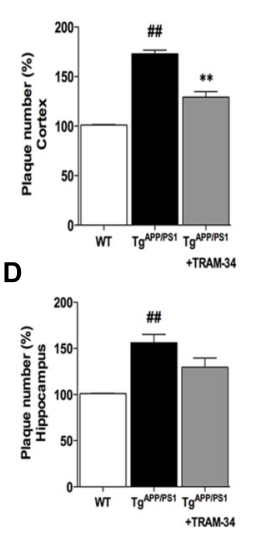

FIGURE 5 | Blockade of KCa3.1 attenuated $\beta$-amyloid burden in the brains of TgAPP/PS1 mice. Mouse anti-6E10 monoclonal antibody was used to detect senile plaques in (A) brain cortex and (C) hippocampus of WT, TgAPP/PS1, and TgAPP/PS1 + TRAM-34 (120 mg/kg) groups $(n=10$ per group). Quantification of senile plaque numbers in (B) cortex and (D) hippocampus. Data represent mean \pm SEM. \#\# $p<0.01$, versus WT mice. ${ }^{* *} p<0.01$, versus vehicle-treated TgAPP/PS1 mice. Scale bar: $50 \mu \mathrm{m}$.

A

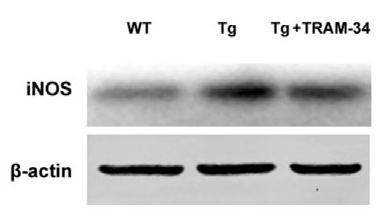

C

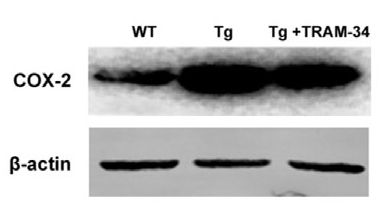

E

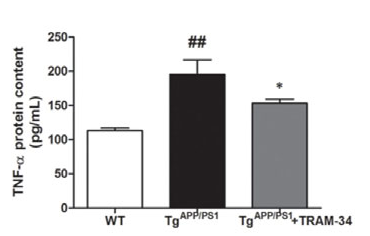

$\mathbf{F}$

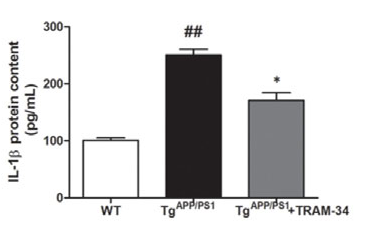

FIGURE 6 | Blockade of $\mathrm{KCa} 3.1$ attenuated expression and release of inflammatory mediators in the brains of $\mathrm{Tg}^{\mathrm{APP} / \mathrm{PS} 1}$ mice. Western blots showing protein expression of $\mathbf{( A , B )}$ iNOS and $\mathbf{( C , D )}$ COX-2 proteins in the brains of WT and TgAPP/PS1 mice, with or without treatment with TRAM-34 $(n=4)$, at the age of 9 months. Measurement of $(\mathbf{E})$ TNF- $\alpha$ and $(\mathbf{F}) \| \mathrm{L}-1 \beta$ released by ELISA in homogenated cortex of WT and TgAPP/PS1 mice, with or without treatment with TRAM-34 $(n=4)$ at the age of 9 months. Data represent mean \pm SEM. ${ }^{\# \#} p<0.01$ compared with WT mice. ${ }^{*} p<0.05$, ${ }^{* *} p<0.01$ compared with TgAPP/PS1 mice.

test, the $\mathrm{Tg}^{\mathrm{APP} / \mathrm{PS} 1}+$ vehicle group showed memory deficits compared with WT mice. The TgAPP/PS1 + TRAM-34 group showed significantly improved spatial learning and memory

compared with the $\mathrm{Tg}^{\mathrm{APP} / \mathrm{PS} 1}+$ vehicle group $(p<0.05$, Figure 3A). In the spatial probe trial without an escape platform, the $\mathrm{Tg}^{\mathrm{APP} / \mathrm{PS} 1}+\mathrm{TRAM}-34$ group spent more time and swam for greater distances in the target quadrant than the $\mathrm{Tg}^{\mathrm{APP} / \mathrm{PS} 1}+$ vehicle group $(p<0.05$, Figures $3 \mathrm{~B}, \mathrm{C})$.

In the open field test, the $\mathrm{Tg}^{\mathrm{APP}} / \mathrm{PS} 1+$ vehicle group had a decreased ratio distance $(p<0.01$, Figure 3D) and ratio time $(p<0.01$, Figure 3E) in the center compared to WT mice. In contrast, the TgAPP/PS1 + TRAM-34 group had a significantly greater ratio distance $(p<0.05$, Figure 3D) and more ratio time $\left(p<0.05\right.$, Figure 3E) in the center than the $\operatorname{Tg}^{\text {APP/PS1 }}+$ vehicle group.

\section{Blockade of KCa3.1 Attenuates Gliosis and Loss of Neurons in TgAPP/PS1 Mice}

Reactive astrogliosis is a common pathological feature of chronic neurological diseases associated with aging, such as AD (Khakh and Sofroniew, 2015). We have previously shown that inhibition of $\mathrm{KCa} 3.1$ reduces the reactive astrogliosis response ( $\mathrm{Yu}$ et al., 2014). In the present study, both $\mathrm{GFAP}^{+}$-reactive astrocytes and $\mathrm{Ibal}^{+}$-activated microglia were significantly increased in the TgAPP/PS1 + vehicle group compared with WT mice. The TgAPP/PS1 + TRAM-34 group, however, showed a significantly suppressed astrogliosis response $(p<0.05$, Figures $4 \mathbf{A}, \mathbf{B})$ and reduced numbers of activated microglia $(p<0.05$, Figures 4C,D) compared with the $\mathrm{Tg}^{\mathrm{APP} / \mathrm{PS} 1}+$ vehicle group. The TgAPP/PS1 + TRAM-34 group also showed reduced neuronal loss in the cortex $(p<0.05$, Figures $4 \mathrm{E}, \mathbf{F})$ and CA1 hippocampus $\left(p<0.05\right.$, Figures 4G,H). Significantly more $\mathrm{NeuN}^{+}$neurons were present in the $\mathrm{Tg}^{\mathrm{APP} / \mathrm{PS} 1}+\mathrm{TRAM}-34$ group than in the $\mathrm{Tg}^{\mathrm{APP} / \mathrm{PS} 1}+$ vehicle group.

Because dendritic and synaptic damage, as well as neuronal loss, likely play important roles in the process of $\mathrm{AD}$, we questioned whether astrogliosis damages dendrites and synapses. 
A

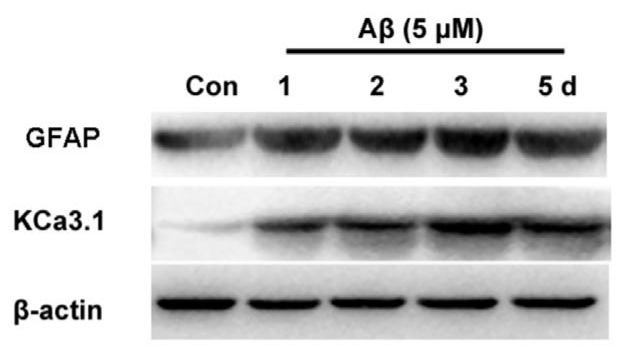

B

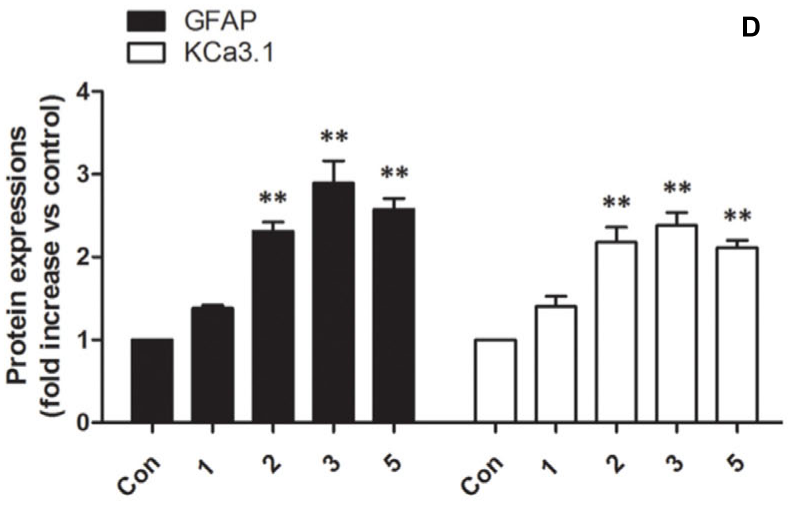

C
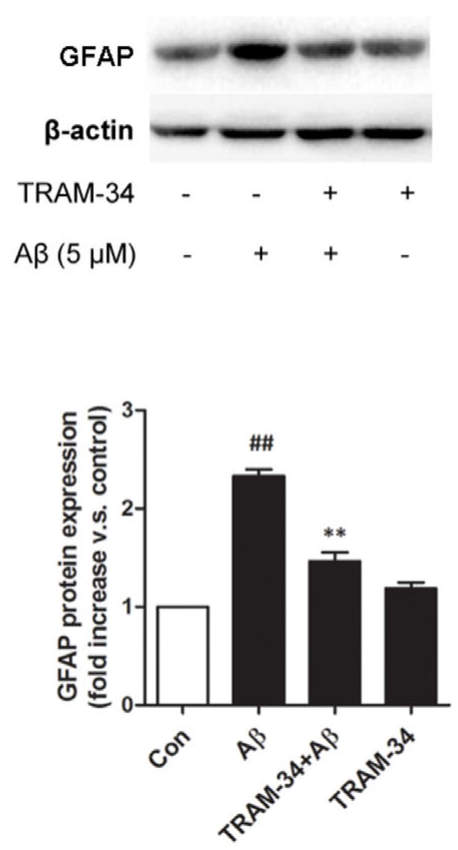

FIGURE 7 | KCa3.1 is involved in A $\mathbf{\beta}$ oligomer-induced astrogliosis. (A) Western blot showing protein expression of astrocytic GFAP and KCa3.1 after treatment with $\mathrm{A} \beta(5 \mu \mathrm{M})$ for $1,2,3$, or 5 days $(n=3)$. (B) Data are presented as mean \pm SEM. ${ }^{* *} p<0.01$ compared with control. (C) Western blot showing expression of GFAP protein after stimulation with A $3(5 \mu \mathrm{M})$, with or without TRAM-34 (1 $\mu \mathrm{M})$ for 3 days $(n=3)$. (D) Data represent mean \pm SEM. ** $p<0.01$ compared with $A \beta$-treatment group; ${ }^{\# \#} p<0.01$ compared with control. Con, control.

Western blotting demonstrated that treatment with TRAM$34(120 \mathrm{mg} / \mathrm{kg})$ significantly reduced levels of astrogliosis and increased expression of dendritic marker MAP2 and excitatory synapses marker PSD95, but not the pre-synaptic marker synaptophysin, in Tg ${ }^{\mathrm{APP} / \mathrm{PS} 1}$ mice (Figures $4 \mathbf{I}, \mathbf{J}$ ).

\section{Blockade of KCa3.1 Decreases Amyloid Deposition and Inflammatory Cytokine Production in Tg ${ }^{A P P} / \mathrm{PS} 1$ Mice}

To investigate whether inhibition of KCa3.1 channels can attenuate amyloid accumulation, cerebral senile plaques in the different groups of mice were visualized using 6E10 staining. Senile plaque formation in both cortex and hippocampus was significantly increased in the $\mathrm{Tg}^{\mathrm{APP} / \mathrm{PS} 1}+$ vehicle group compared with WT mice $(p<0.01$, Figures 5A-D). After treatment with TRAM-34 (120 mg/kg, i.p.) for 4 weeks, Tg APP/PS1 mice showed decreased senile plaque formation. Quantification showed that senile plaque formation was decreased in the cortex $(p<0.01$; Figure 5B) but not in the hippocampus $(p>0.05$; Figure 5D) in the $\mathrm{Tg}^{\mathrm{APP} / \mathrm{PS} 1}+\mathrm{TRAM}-34$ group compared with the Tg ${ }^{\mathrm{APP}} / \mathrm{PS} 1+$ vehicle group. Given the contribution of gliosis to $\mathrm{A} \beta$ production, the decrease in senile plaque formation seen in the cortex of $\mathrm{Tg}^{\mathrm{APP} / \mathrm{PS} 1}+\mathrm{TRAM}-34$ group is likely attributable to a reduction in numbers of reactive astrocytes and active microglia, brought about by inhibition of KCa3.1 channels.
Neuronal death in $\mathrm{AD}$ is largely caused by the release of pro-inflammatory mediators during gliosis (Scuderi et al., 2014). Blockade of KCa3.1 inhibited the up-regulation of iNOS (Figures 6A,B) and COX-2 (Figures 6C,D), compared with vehicle-treated $\mathrm{Tg}^{\mathrm{APP} / \mathrm{PS} 1}$ mice. Levels of IL- $1 \beta$ and TNF- $\alpha$ in brain homogenates from the different groups were measured by ELISA experiments (Figures 6E,F). Levels of both IL-1 $\beta$ and TNF- $\alpha$ released were attenuated in the TgAPP/PS1 + TRAM-34 group compared with the Tg ${ }^{\mathrm{APP} / \mathrm{PS} 1}+$ vehicle group. These data demonstrate that KCa3.1 is involved in activation of glial cells in a mouse model of AD.

\section{Blockade of KCa3.1 Attenuated A $\beta$-Induced Reactive Astrogliosis through JNK Signaling Pathways}

We first tested whether $A \beta$-induced astrogliosis was associated with KCa3.1 expression in vitro. Primary astrocytes cultured in the presence of $\mathrm{A} \beta(5 \mu \mathrm{M})$ showed up-regulated GFAP and KCa3.1 (Figures 7A,B). The up-regulation of GFAP was blocked by pre-treatment with TRAM-34 (1 $\mu \mathrm{M}$; Figures 7C,D). The concentration of TRAM-34 (1 $\mu \mathrm{M})$ was used as previously described (Bouhy et al., 2011; Yu et al., 2014).

Reactive astrocytes release a variety of cytokines and proinflammatory mediators, which activate intracellular MAPK signaling pathways (Matos et al., 2008). We examined whether the involvement of KCa3.1 in $\mathrm{A} \beta$-induced astrogliosis is mediated 

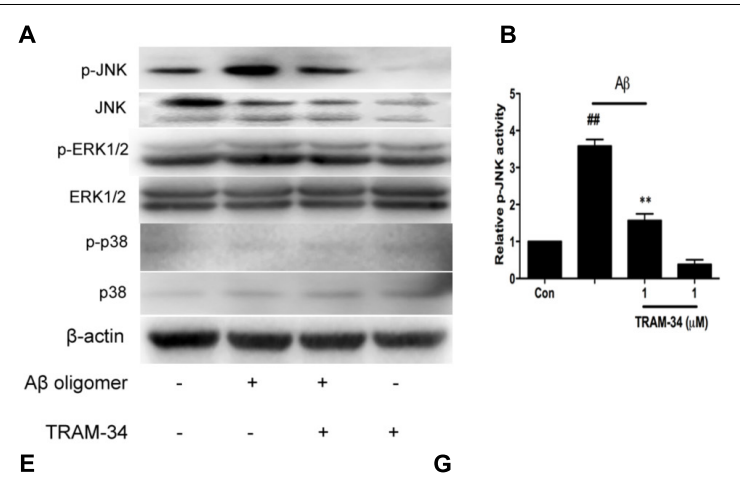

$\circ$
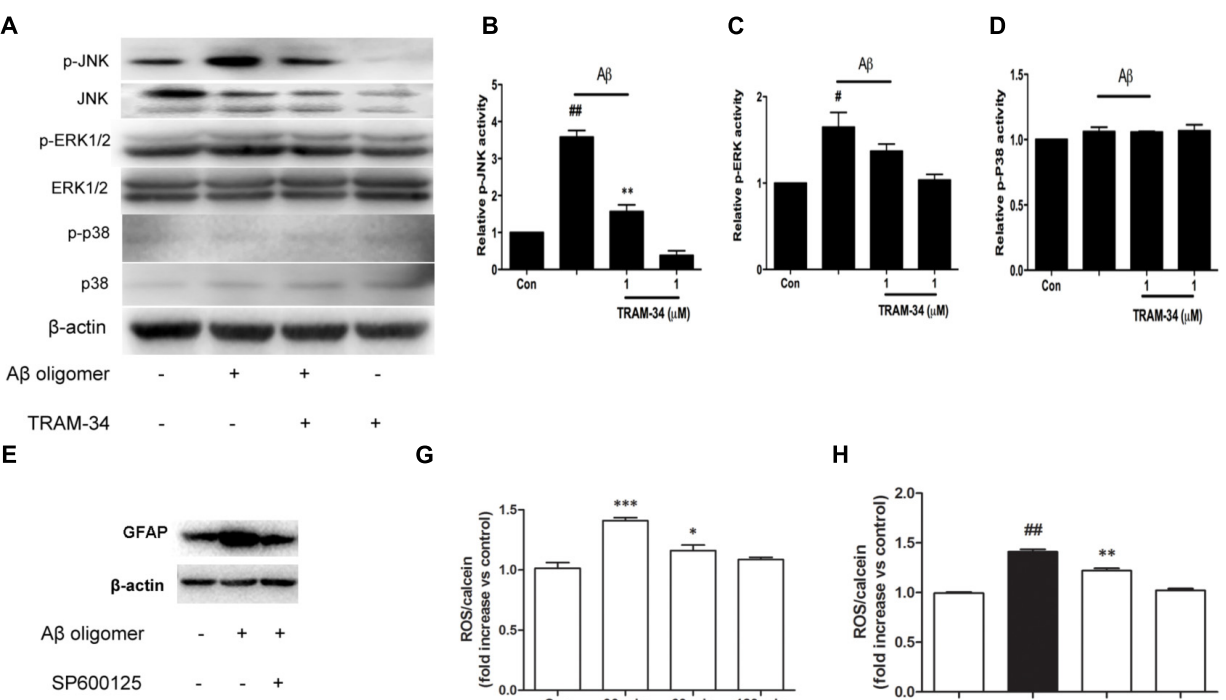

$\mathbf{F}$

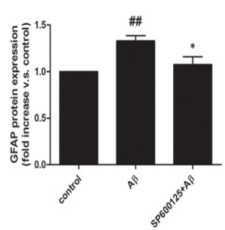

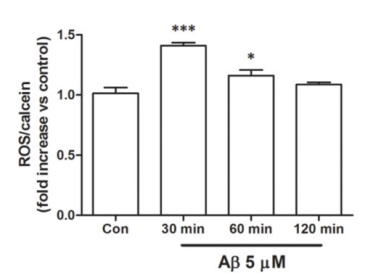

H

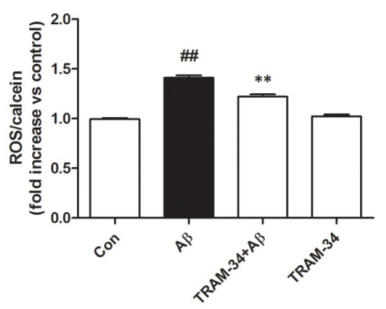

FIGURE 8 | KCa3.1 is involved in A $\boldsymbol{\beta}$-induced generation of activated astrocyte phenotype. (A) Representative images of total JNK/ERK/P38 and $\mathrm{p}-\mathrm{JNK} / \mathrm{p}-\mathrm{ERK} / \mathrm{p}-\mathrm{P} 38$, in control astrocytes and A $\beta$-stimulated astrocytes (30 min), with or without TRAM-34 (1 $\mu \mathrm{M})$ treatment. (B-D) Mean values of p-JNK/p-ERK/p-P38 activity relative to total JNK/ERK/P38 $(n=3-6)$. Results are presented as mean \pm SEM. ${ }^{\#} p<0.05,{ }^{\# \#} p<0.01$ versus control, ${ }^{* *} p<0.01$ versus $A \beta$-stimulated alone. (E,F) Western blots showing expression of GFAP protein in astrocytes after 3 days A $\beta$ stimulation in the presence of SP600125 (10 $\mu$ M; $n=3$ ). Data are presented as mean \pm SEM. ${ }^{\# \#} p<0.01$ versus control, ${ }^{*} p<0.05$ versus A $\beta$. (G) CM-H2DCFDA (30 $\mu$ M) was used to measure the level of reactive oxygen species (ROS) generation induced by A $\beta$ in astrocytes. (H) ROS generation was reduced by TRAM-34 $(1 \mu \mathrm{M})$ in astrocytes treated with A $\beta$ ( $n=5)$. Data represent mean \pm SEM. $\beta$-actin was used as an internal control. ${ }^{\# \#} p<0.01,{ }^{* * *} p<0.001,{ }^{*} p<0.05$ compared with control; ${ }^{* *} p<0.01$ compared with $A \beta$-treated cells.

by the ERK, JNK, and p38 signaling pathways (Figures 8A-D). Astrocytes exposed to $\mathrm{A} \beta(5 \mu \mathrm{M})$ for $30 \mathrm{~min}$ showed upregulated phosphorylation of JNK (Figures 8A,B) and ERK (Figures 8A,C) but only phosphorylation of JNK was inhibited by TRAM-34 (1 $\mu$ M; Figure 8B). Treatment with the JNK inhibitor SP600125 $(10 \mu \mathrm{M})$ for 3 days attenuated A $\beta$-induced GFAP protein expression in astrocytes (Figures $\mathbf{8 E}, \mathbf{F}$ ).

The molecular probe $\mathrm{CM}-\mathrm{H}_{2} \mathrm{DCFDA}$, which detects intracellular ROS levels, was used to determine whether KCa3.1 contributed to ROS production via $\mathrm{A} \beta$-induced astrogliosis. After treatment with $\mathrm{A} \beta$ oligomer $(5 \mu \mathrm{M})$ for $30 \mathrm{~min}$, a rapid transient increase in the DCF signal was detected (Figure 8G). ROS production at 30 min was inhibited by TRAM-34 $(p<0.01$, Figure 8H). These data demonstrate that KCa3.1 is involved in reactive astrocytosis and oxidative stress via the JNK signaling cascades.

\section{Blockade of KCa3.1 Attenuated Indirect $A \beta$-Induced Neurotoxicity Mediated by Astrocytes}

It has been reported that astrocytes accelerate $A \beta$-induced neurotoxicity (Garwood et al., 2011). To determine whether or not $\mathrm{KCa} 3.1$ is involved in astrocyte-accelerated $\mathrm{A} \beta$-induced neurotoxicity, neurons, astrocytes and mixed cultures (neurons with astrocytes) were treated with $5 \mu \mathrm{M} A \beta$ oligomers and then cell viability was measured in the different groups at 48 or $72 \mathrm{~h}$ (Figures 9A-E). Treatment with $1 \mu \mathrm{M}$ TRAM-34 alone did not significantly change the viability of any of these cell cultures. Pre-treatment of mixed cultures, but not neuronal cultures, with TRAM-34 before treatment with $\mathrm{A} \beta$ increased cell viability at both 48 and $72 \mathrm{~h}$ (Figures 9A,C), suggesting that TRAM-34 might reduce $A \beta$-induced neurotoxicity by suppressing astrogliosis. A significant decrease in cell viability was observed only after $72 \mathrm{~h}$ in $\mathrm{A} \beta$-treated neuronal cultures (Figures 9B,D). A $\beta$ oligomers did not cause decreased viability from astrocytes at $72 \mathrm{~h}$ (Figure 9E), indicating that the phenotype switch of astrogliosis significantly accelerates $A \beta$-induced neurotoxicity.

We next asked whether blockade of KCa3.1 might attenuate neurotoxicity induced by inflammatory mediators during astrogliosis. Pre-treatment with TRAM-34 (1 $\mu \mathrm{M})$ was found to rescue neuronal viability in cultures treated with astrocytes CM (Figure 10A). As a negative control, neuronal viability was not significantly affected by cell-free astrocyte CM that had been incubated for $48 \mathrm{~h}$ with $\mathrm{A} \beta$ ( $5 \mu \mathrm{M}$; Figure 10B). 
A

C
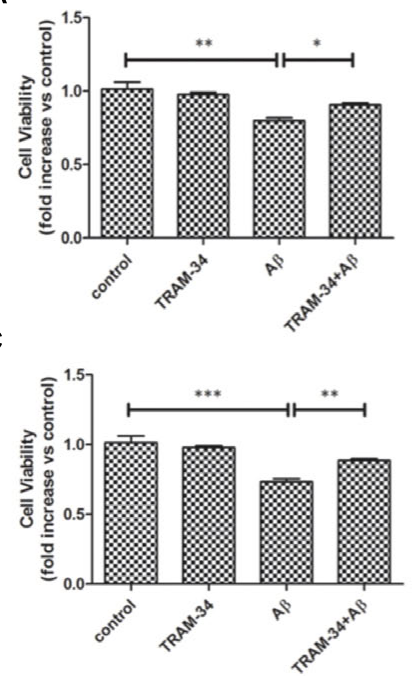

B
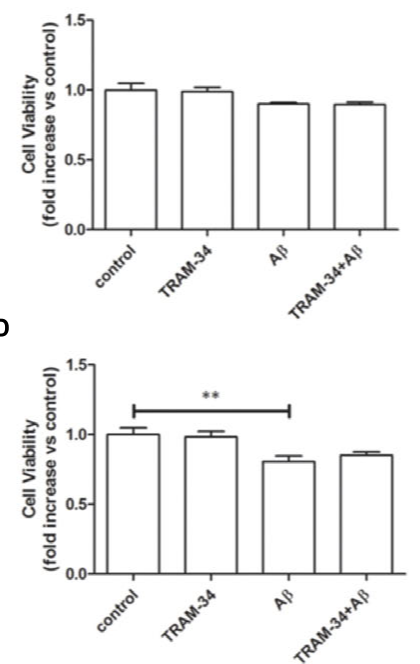

E

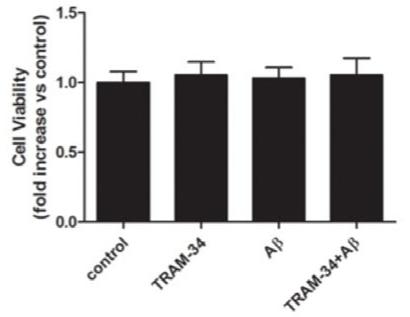

FIGURE 9 | KCa3.1 is involved in astrocyte-accelerated $\mathbf{A} \boldsymbol{\beta}$-induced neurotoxicity. Bar charts showing cell viability of (A,C) mouse primary mixed cultures and $\mathbf{( B , D )}$ neuronal cultures treated with $\mathrm{A \beta}(5 \mu \mathrm{M})$ for $\mathbf{( A , B )} 48$ or $(\mathbf{C}, \mathbf{D}) 72 \mathrm{~h}$, with or without pre-treatment with TRAM-34 (1 $\mu \mathrm{M})$. (E) Cell viability of mouse primary astrocytes treated for $72 \mathrm{~h}$ with $\mathrm{A} \beta(5 \mu \mathrm{M})$, with and without pre-treatment with TRAM-34 $(1 \mu \mathrm{M})$. Data represent mean $\pm \mathrm{SEM}$ of cell viability relative to control ( $n=4$ cultures). ${ }^{*} p<0.05,{ }^{* *} p<0.01,{ }^{* * *} p<0.001$

A

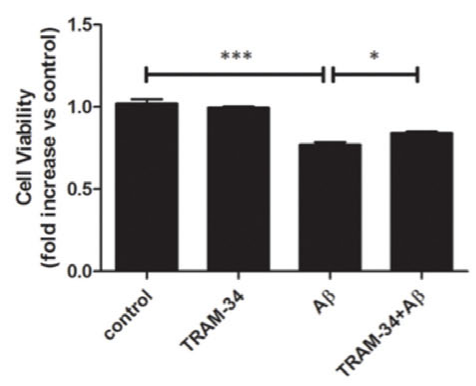

B

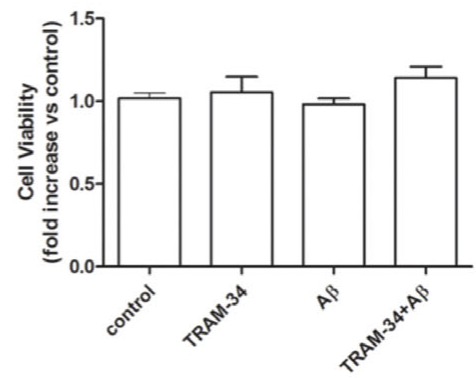

FIGURE 10 | A $\beta$-induced indirect, astrogliosis-mediated neurotoxicity. Bar charts showed cell viability of neurons exposed to (A) CM from astrocytes treated with A $\beta(5 \mu \mathrm{M})$, with or without TRAM-34 (1 $\mu \mathrm{M})$ and (B) cell-free astrocytes culture medium treated with A $(5 \mu \mathrm{M})$ for $48 \mathrm{~h}$, with or without TRAM-34 (1 $\mu \mathrm{M})$. Data represent mean $\pm \mathrm{SEM}$ of cell viability relative to control $\left(n=4\right.$ cultures). ${ }^{*} p<0.05,{ }^{* * *} p<0.001$. CM, conditioned medium.

Because damage to dendrites and synapses occurs before neuronal loss during the development of AD (Szegedi et al., 2006), we asked whether $A \beta$-induced astrogliosis-mediated by KCa3.1 could accelerate damage to dendrites and synapses. More signs of dendritic and synaptic damage were observed in neurons treated with $A \beta$-treated astrocytes $C M$ than in those treated with control CM, as demonstrated by immunofluorescent staining for the dendritic marker MAP2 (Figure 11A). Incubation with $\mathrm{A} \beta$-CM decreased total neurite length (Figure 11B) and number of branch points (Figure 11C). Pre-incubation with TRAM-34 ( 1 and $10 \mu \mathrm{M}$ ) markedly reversed the effect of $\mathrm{A} \beta$-CM by increasing total neurite length and number of branch points (Figures 11A-C). The concentrations of TRAM-34 (1 and $10 \mu \mathrm{M}$ ) were used as previously described (Lallet-Daher et al., 2009; Yu et al., 2014). Western blots showed that blockade of KCa3.1 attenuated A $\beta$-CM-induced decreases in NeuN,
MAP2, and PSD95, but not decreases in the pre-synaptic marker, synaptophysin (Figures 11D,E). Taken together, these results show that $\mathrm{KCa} 3.1$ is involved in $\mathrm{A} \beta$-mediated damage to dendrites and synapses by an indirect, astrogliosis-mediated mechanism.

\section{DISCUSSION}

The data presented herein demonstrate that blockade of KCa3.1 attenuates neuropathology by regulating neuroinflammation in a mouse model of $\mathrm{AD}$ and, moreover, that prevention of astrogliosis might be a promising strategy for the treatment of $\mathrm{AD}$. Using $\mathrm{Tg}{ }^{\mathrm{APP}} / \mathrm{PS} 1$ mice as a model of $\mathrm{AD}$, we have shown that pharmacological blockade of KCa3.1 significantly reduced astrogliosis, neuronal loss, and memory deficits. KCa3.1 blockade 


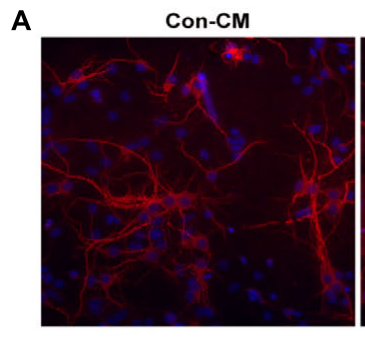

B

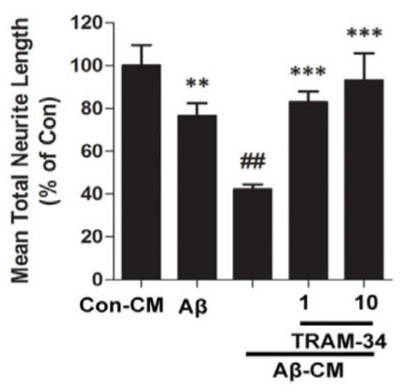

D

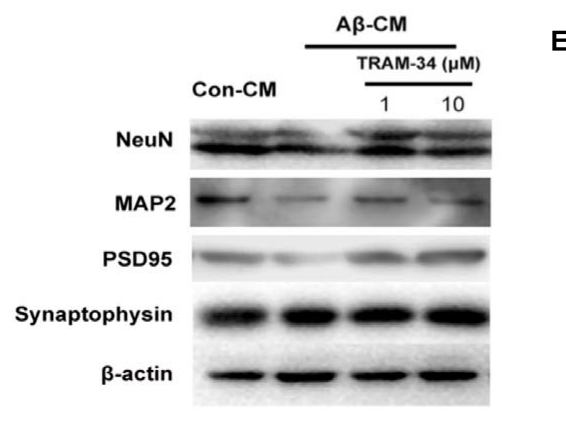

$A \beta$

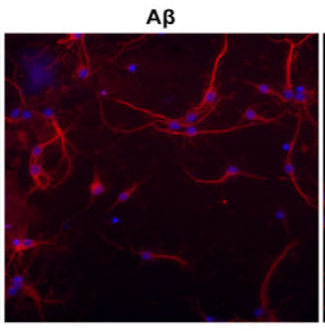

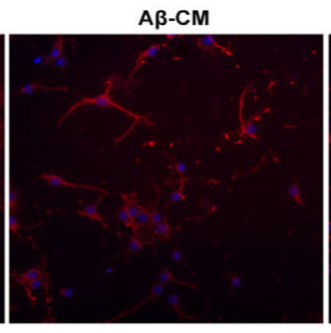

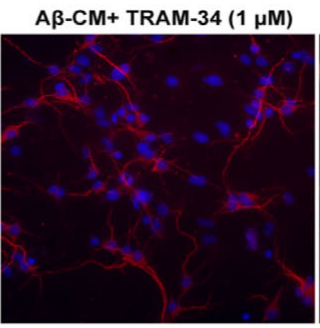

C

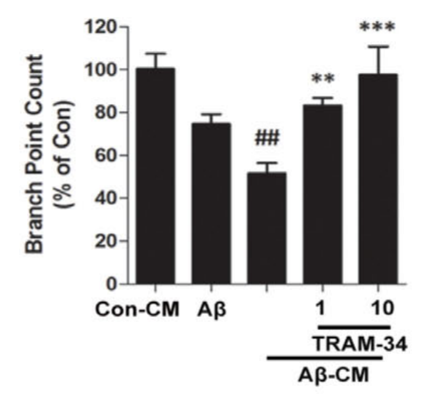

E

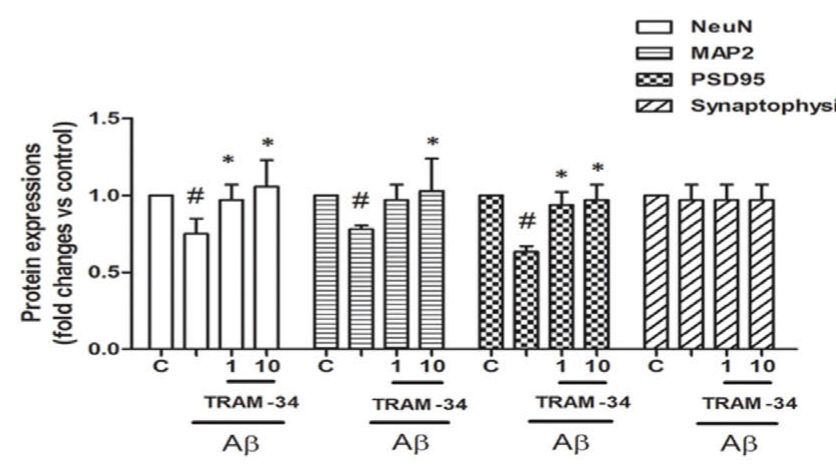

FIGURE 11 | Blockade of KCa3.1 attenuated A $\beta$-induced, indirect, astrocyte-mediated damage to dendrites and synapses. Levels of dendritic and synaptic markers were compared between hippocampal neurons treated with solvent only (control treatment), $5 \mu \mathrm{M} A \beta, \mathrm{A} \beta$-CM, or $\mathrm{CM}$ from astrocyte cultures in which $A \beta$-induced activation was inhibited for 24 h by TRAM-34 (TRAM-34 + A $\beta$-CM). A Cellomics KineticScan HCS Reader was used to image the primary cerebral neurons. (A) For better visualization of individual dendrites, dendrites of the sparsely plated neurons were immunostained with MAP2 (red), and nuclei were stained with DAPI (blue). Extended Neurite Outgrowth bioapplication software was used to analyze (B) neurite length and (C) branch point counts. Data represent mean $\pm \operatorname{SEM}(n=3) .{ }^{* *} p<0.01,{ }^{* * *} p<0.001$ compared with A $\beta$-CM. ${ }^{\# \#} p<0.01$ compared with control. A $\beta$-CM treatment significantly reduced neurite length and branch point counts. This reduction was prevented by inhibition of astrocyte activation by TRAM-34 (1 and $10 \mu \mathrm{M}$; $n=3)$. (D) Western blot analysis with the following primary antibodies: neuronal marker NeuN, dendritic marker MAP2, post-synaptic proteins PSD95, and pre-synaptic protein synaptophysin. (E) Results are presented as mean $\pm \operatorname{SEM}(n=3) .{ }^{*} p<0.05$ compared with con-CM. ${ }^{*} p<0.05$ compared with A $\beta$-CM. CM, conditioned medium.

inhibited astrocyte activation and reduced levels of IL-1 $\beta$, TNF$\alpha$, iNOS, and COX-2 in the brain. In vitro experiments with primary murine neuronal, astrocytic and mixed cortical cultures exposed to $\mathrm{A} \beta$ confirmed that blockade of $\mathrm{KCa} 3.1$ improved neuronal survival by reducing astrocyte activation and ROS production.

Deposits of $\mathrm{A} \beta$ could induce oxidative stress, glial activation, and local cell loss. In the progression of $\mathrm{AD}$, astrocytes undergo a switch to a reactive phenotype, characterized by profound functional and morphological alterations (O'Callaghan et al., 2014). Up-regulation of the astrogliosis marker GFAP is a wellcharacterized example of these phenotypical changes and, in our cellular model, TRAM-34 was found to negatively modulate expression of both GFAP and KCa3.1.

There is a large body of data that implicates MAPK signaling pathways, especially JNK pathways, in oxidative stress (Matos et al., 2008). In the present study, blockade of KCa3.1 attenuated phosphorylation of JNK, which is activated by $\mathrm{A} \beta$ stimulation. Astrogliosis has been shown to contribute to inflammatory processes in $\mathrm{AD}$ through the release of ROS, pro-inflammatory factors, and cytokines (Heneka et al., 2001; Verkhratsky et al., 2010). For example, IL-1 $\beta$, TNF- $\alpha$, ROS, and NO have been found to regulate pro- and anti-inflammatory genes, including NOS-2 and COX-2 (Tuppo and Arias, 2005; Wyss-Coray, 2006). 
We found that blockade of KCa3.1 attenuated $\mathrm{A} \beta$-induced indirect neurotoxicity and decreased $\mathrm{A} \beta$-mediated damage to dendrites and synapses by an indirect, astrogliosis-mediated mechanism. In the AD mouse model, levels of pro-inflammatory factors and cytokines were significantly up-regulated and these increases were inhibited by TRAM-34.

The tripartite synapses in the CNS are formed by pre- and post-synaptic neuronal compartments and astroglial perisynaptic processes (Verkhratsky et al., 2010). A phenotype switch of astrocytes may lead to abnormal release of gliotransmitters, such as glutamate and GABA, which could lead to synaptic loss, excitotoxicity, and neurodegeneration in $\mathrm{AD}$ (Agulhon et al., 2012; Jo et al., 2014). Numerous clinical studies have confirmed a correlation between the degree of dementia and the extent of synaptic loss. In our studies, blockade of KCa3.1 rescued memory deficits, and synapse/neuron loss in $\mathrm{Tg}^{\mathrm{APP} / \mathrm{PS} 1}$ mice. After treatment with TRAM-34, quantification showed that senile plaque formation was decreased in the cortex (Figure 5B) but not in the hippocampus (Figure 5D). We propose that the different efficacy with respect to different brain areas is that the hippocampus was the greatest amyloid burden region in mice model of AD (Reilly et al., 2003), and the processes of astrogliosis mostly observed surrounding amyloid plaques also were able to accumulate large amounts of senile plaque (Rodriguez et al., 2009). Reducing astrogliosis is a promising strategy for controlling harmful CNS inflammation in neurodegenerative disorders (Khakh and Sofroniew, 2015; Sofroniew, 2015).

In the present in vivo study, we found that KCa3.1 was up-regulated in reactive astrocytes of $\mathrm{Tg}^{\mathrm{APP} / \mathrm{PS} 1}$ mice and $\mathrm{AD}$ patients compared with WT mice and control humans. It has also been reported that $\mathrm{KCa} 3.1$ is involved in several aspects of microglial activation in vitro (Kaushal et al., 2007; Maezawa et al., 2011; Ferreira and Schlichter, 2013). Maezawa et al. (2011) reported that KCa3.1 activity is required for $\mathrm{A} \beta$-induced microglial neurotoxicity in vitro. In our study, KCa3.1 channels were rarely co-localized with microglia, although blockade of $\mathrm{KCa} 3.1$ reduced both astrogliosis and microglial activation in $\mathrm{Tg}^{\mathrm{APP} / \mathrm{PS} 1}$ mice.

\section{REFERENCES}

Abramov, A. Y., Canevari, L., and Duchen, M. R. (2003). Changes in intracellular calcium and glutathione in astrocytes as the primary mechanism of amyloid neurotoxicity. J. Neurosci. 23, 5088-5095.

Agulhon, C., Sun, M. Y., Murphy, T., Myers, T., Lauderdale, K., and Fiacco, T. A. (2012). Calcium signaling and gliotransmission in normal vs. reactive astrocytes. Front. Pharmacol. 3:139. doi: 10.3389/fphar.2012.00139

Alberdi, E., Wyssenbach, A., Alberdi, M., Sanchez-Gomez, M. V., Cavaliere, F., Rodriguez, J. J., et al. (2013). Ca(2+) -dependent endoplasmic reticulum stress correlates with astrogliosis in oligomeric amyloid beta-treated astrocytes and in a model of Alzheimer's disease. Aging Cell 12, 292-302. doi: 10.1111/acel.12054

Bouhy, D., Ghasemlou, N., Lively, S., Redensek, A., Rathore, K. I., Schlichter, L. C., et al. (2011). Inhibition of the $\mathrm{Ca}(2)(+)$-dependent $\mathrm{K}(+)$ channel, $\mathrm{KCNN} 4 / \mathrm{KCa} 3.1$, improves tissue protection and locomotor recovery after spinal cord injury. J. Neurosci. 31, 16298-16308. doi: 10.1523/JNEUROSCI. 0047-11.2011

Carter, S. F., Schöll, M., Almkvist, O., Wall, A., Engler, H., Långström, B., et al. (2012). Evidence for astrocytosis in prodromal Alzheimer disease provided
Based on these results, we propose that the expression of $\mathrm{KCa} 3.1$ is under the immunohistochemically detectable levels in microglia of both mice and human. Co-cultures of astrocytes and neurons were established to determine whether or not KCa3.1 is involved in astrocyte-accelerated $A \beta$-induced neurotoxicity. Our data suggest that TRAM34 might reduce $A \beta$-induced neurotoxicity by suppressing astrogliosis.

The mechanism of the $A \beta$-induced phenotypic switch of astrocytes and its potential role in the progression of $\mathrm{AD}$ provide excellent opportunities for novel therapies such as TRAM-34, a small molecule blocker of KCa3.1. We have provided evidence that KCa3.1 regulates $\mathrm{A} \beta$-induced astrogliosis and neurotoxicity. The anti-inflammatory and neuroprotective properties of KCa3.1 blockers have been demonstrated in many animal models, including traumatic brain injury, retinal ganglion cell degeneration, and multiple sclerosis (Mauler et al., 2004; Reich et al., 2005; Kaushal et al., 2007; Yi et al., 2016b). Our data show that KCa3.1 is also a promising target for reducing inflammatory damage in AD.

\section{AUTHOR CONTRIBUTIONS}

ZY supervised the entire project, designed research, and wrote the paper. HC conceived and designed the experiments, interpreted and analyzed data, supervised all the experimental procedure. TW and MY conceived and designed the experiments, performed research interpreted and analyzed data. QL performed research and analyzed data. WG and LH analyzed data and critically revised the manuscript.

\section{FUNDING}

This work was supported by Science and Technology Commission of Shanghai Municipality grant 16ZR1418700, National Natural Science Foundation of China grant 81503044.

by $11 \mathrm{C}$-deuterium-L-deprenyl: a multitracer PET paradigm combining 11CPittsburgh compound B and 18F-FDG. J. Nucl. Med. 53, 37-46. doi: 10.2967/ jnumed.110.087031

Chen, C. L., Liao, J. W., Hu, O. Y., and Pao, L. H. (2016). Blockade of KCa3.1 potassium channels protects against cisplatin-induced acute kidney injury. Arch. Toxicol. 90, 2249-2260. doi: 10.1007/s00204-015-1607-5

Di, L., Srivastava, S., Zhdanova, O., Ding, Y., Li, Z., Wulff, H., et al. (2010). Inhibition of the $\mathrm{K}+$ channel KCa3.1 ameliorates $\mathrm{T}$ cell-mediated colitis. Proc. Natl. Acad. Sci. U.S.A. 107, 1541-1546. doi: 10.1073/pnas.0910133107

Ferreira, R., and Schlichter, L. C. (2013). Selective activation of KCa3.1 and CRAC channels by $\mathrm{P} 2 \mathrm{Y} 2$ receptors promotes $\mathrm{Ca}(2+)$ signaling, store refilling and migration of rat microglial cells. PLoS ONE 8:e62345. doi: 10.1371/journal.pone. 0062345

Garwood, C. J., Pooler, A. M., Atherton, J., Hanger, D. P., and Noble, W. (2011). Astrocytes are important mediators of Abeta-induced neurotoxicity and tau phosphorylation in primary culture. Cell Death Dis. 2:e167. doi: 10.1038/cddis. 2011.50

Heneka, M. T., Wiesinger, H., Dumitrescu-Ozimek, L., Riederer, P., Feinstein, D. L., and Klockgether, T. (2001). Neuronal and glial coexpression of 
argininosuccinate synthetase and inducible nitric oxide synthase in Alzheimer disease. J. Neuropathol. Exp. Neurol. 60, 906-916. doi: 10.1093/jnen/60.9.906

Jo, S., Yarishkin, O., Hwang, Y. J., Chun, Y. E., Park, M., Woo, D. H., et al. (2014). GABA from reactive astrocytes impairs memory in mouse models of Alzheimer's disease. Nat. Med. 20, 886-896. doi: 10.1038/nm.3639

Kaushal, V., Koeberle, P. D., Wang, Y., and Schlichter, L. C. (2007). The Ca2+activated $\mathrm{K}+$ channel $\mathrm{KCNN} 4 / \mathrm{KCa} 3.1$ contributes to microglia activation and nitric oxide-dependent neurodegeneration. J. Neurosci. 27, 234-244. doi: 10. 1523/JNEUROSCI.3593-06.2007

Khakh, B. S., and Sofroniew, M. V. (2015). Diversity of astrocyte functions and phenotypes in neural circuits. Nat. Neurosci. 18, 942-952. doi: 10.1038/nn.4043

Lallet-Daher, H., Roudbaraki, M., Bavencoffe, A., Mariot, P., Gackière, F., Bidaux, G., et al. (2009). Intermediate-conductance $\mathrm{Ca} 2+$-activated $\mathrm{K}+$ channels (IKCa1) regulate human prostate cancer cell proliferation through a close control of calcium entry. Oncogene 28, 1792-1806. doi: 10.1038/onc. 2009.25

Maezawa, I., Zimin, P. I., Wulff, H., and Jin, L. W. (2011). Amyloid-beta protein oligomer at low nanomolar concentrations activates microglia and induces microglial neurotoxicity. J. Biol. Chem. 286, 3693-3706. doi: 10.1074/jbc.M110. 135244

Matos, M., Augusto, E., Oliveira, C. R., and Agostinho, P. (2008). Amyloidbeta peptide decreases glutamate uptake in cultured astrocytes: involvement of oxidative stress and mitogen-activated protein kinase cascades. Neuroscience 156, 898-910. doi: 10.1016/j.neuroscience.2008.08.022

Mauler, F., Hinz, V., Horvath, E., Schuhmacher, J., Hofmann, H. A., Wirtz, S., et al. (2004). Selective intermediate-/small-conductance calcium-activated potassium channel (KCNN4) blockers are potent and effective therapeutics in experimental brain oedema and traumatic brain injury caused by acute subdural haematoma. Eur. J. Neurosci. 20, 1761-1768. doi: 10.1111/j.1460-9568.2004. 03615.x

Morris, R. (1984). Developments of a water-maze procedure for studying spatial learning in the rat. J. Neurosci. Methods 11, 47-60. doi: 10.1016/0165-0270(84) 90007-4

O'Callaghan, J. P., Kelly, K. A., VanGilder, R. L., Sofroniew, M. V., and Miller, D. B. (2014). Early activation of STAT3 regulates reactive astrogliosis induced by diverse forms of neurotoxicity. PLOS ONE 9:e102003. doi: 10.1371/journal. pone.0102003

Reich, E. P., Cui, L., Yang, L., Pugliese-Sivo, C., Golovko, A., Petro, M., et al. (2005). Blocking ion channel KCNN4 alleviates the symptoms of experimental autoimmune encephalomyelitis in mice. Eur. J. Immunol. 35, 1027-1036. doi: 10.1002/eji.200425954

Reilly, J. F., Games, D., Rydel, R. E., Freedman, S., Schenk, D., Young, W. G., et al. (2003). Amyloid deposition in the hippocampus and entorhinal cortex: quantitative analysis of a transgenic mouse model. Proc. Natl. Acad. Sci. U.S.A. 100, 4837-4842. doi: 10.1073/pnas.0330745100

Rodriguez, J. J., Olabarria, M., Chvatal, A., and Verkhratsky, A. (2009). Astroglia in dementia and Alzheimer's disease. Cell Death. Differ. 16, 378-385. doi: 10.1038/ cdd.2008.172

Rodriguez-Gonzalez, R., Sobrino, T., Veiga, S., Lopez, P., Rodriguez-Garcia, J., del Rio, S. V., et al. (2016). Neuroprotective effects of dexmedetomidine conditioning strategies: evidences from an in vitro model of cerebral ischemia. Life Sci. 144, 162-169. doi: 10.1016/j.lfs.2015.12.007

Schöll, M., Carter, S. F., Westman, E., Rodriguez-Vieitez, E., Almkvist, O., Thordardottir, S., et al. (2015). Early astrocytosis in autosomal dominant Alzheimer's disease measured in vivo by multi-tracer positron emission tomography. Sci. Rep. 10:16404. doi: 10.1038/srep16404
Scuderi, C., Stecca, C., Valenza, M., Ratano, P., Bronzuoli, M. R., Bartoli, S., et al. (2014). Palmitoylethanolamide controls reactive gliosis and exerts neuroprotective functions in a rat model of Alzheimer's disease. Cell Death Dis. 5:e1419. doi: 10.1038/cddis.2014.376

Sofroniew, M. V. (2015). Astrogliosis. Cold Spring Harb. Perspect. Biol. 7:a020420. doi: 10.1101/cshperspect.a020420

Szegedi, V., Juhasz, G., Rozsa, E., Juhasz-Vedres, G., Datki, Z., Fulop, L., et al. (2006). Endomorphin-2, an endogenous tetrapeptide, protects against Abetal42 in vitro and in vivo. FASEB. J. 20, 1191-1193.

Toyama, K., Wulff, H., Chandy, K. G., Azam, P., Raman, G., Saito, T., et al. (2008). The intermediate-conductance calcium-activated potassium channel KCa3.1 contributes to atherogenesis in mice and humans. J. Clin. Invest. 118, 3025-3037. doi: 10.1172/JCI30836

Tuppo, E. E., and Arias, H. R. (2005). The role of inflammation in Alzheimer's disease. Int. J. Biochem. Cell Biol. 37, 289-305. doi: 10.1016/j.biocel.2004.07.009

Verkhratsky, A., Olabarria, M., Noristani, H. N., Yeh, C. Y., and Rodriguez, J. J. (2010). Astrocytes in Alzheimer's disease. Neurotherapeutics 7, 399-412. doi: 10.1016/j.nurt.2010.05.017

Wang, H., Katagiri, Y., McCann, T. E., Unsworth, E., Goldsmith, P., Yu, Z. X., et al. (2008). Chondroitin-4-sulfation negatively regulates axonal guidance and growth. J. Cell Sci. 121, 3083-3091. doi: 10.1242/jcs.032649

Wang, Y., Xia, Z., Xu, J. R., Wang, Y. X., Hou, L. N., Qiu, Y., et al. (2012). Alpha-mangostin, a polyphenolic xanthone derivative from mangosteen, attenuates beta-amyloid oligomers-induced neurotoxicity by inhibiting amyloid aggregation. Neuropharmacology 62, 871-881. doi: 10.1016/j.neuropharm.2011.09.016

Wu, K., Gao, X., Shi, B., Chen, S., Zhou, X., Li, Z., et al. (2016). Enriched endogenous n-3 polyunsaturated fatty acids alleviate cognitive and behavioral deficits in a mice model of Alzheimer's disease. Neuroscience 333, 345-355. doi: 10.1016/j.neuroscience.2016.07.038

Wyss-Coray, T. (2006). Inflammation in Alzheimer disease: driving force, bystander or beneficial response? Nat. Med. 12, 1005-1015.

Yi, M., Dou, F., Lu, Q., Yu, Z., and Chen, H. (2016a). Activation of the KCa3.1 channel contributes to traumatic scratch injury-induced reactive astrogliosis through the JNK/c-Jun signaling pathway. Neurosci. Lett. 624, 62-71. doi: 10. 1016/j.neulet.2016.05.004

Yi, M., Yu, P., Lu, Q., Geller, H. M., Yu, Z., and Chen, H. (2016b). KCa3.1 constitutes a pharmacological target for astrogliosis associated with Alzheimer's disease. Mol. Cell Neurosci. 76, 21-32. doi: 10.1016/j.mcn.2016.08.008

Yu, Z., Yu, P., Chen, H., and Geller, H. M. (2014). Targeted inhibition of KCa3.1 attenuates TGF-beta-induced reactive astrogliosis through the Smad2/3 signaling pathway. J. Neurochem. 130, 41-49. doi: 10.1111/jnc.12710

Yu, Z. H., Wang, Y. X., Song, Y., Lu, H. Z., Hou, L. N., Cui, Y. Y., et al. (2013). Upregulation of KCa3.1 promotes human airway smooth muscle cell phenotypic modulation. Pharmacol. Res. 77, 30-38. doi: 10.1016/j.phrs.2013.09.002

Conflict of Interest Statement: The authors declare that the research was conducted in the absence of any commercial or financial relationships that could be construed as a potential conflict of interest.

Copyright (c) 2017 Wei, Yi, Gu, Hou, Lu, Yu and Chen. This is an open-access article distributed under the terms of the Creative Commons Attribution License (CC BY). The use, distribution or reproduction in other forums is permitted, provided the original author(s) or licensor are credited and that the original publication in this journal is cited, in accordance with accepted academic practice. No use, distribution or reproduction is permitted which does not comply with these terms. 